\title{
Optical properties of silicene, $\mathrm{Si} / \mathrm{Ag}(111)$, and $\mathrm{Si} / \mathrm{Ag}(110)$
}

\author{
C. Hogan,${ }^{1,2, *}$ O. Pulci, ${ }^{2,3, \dagger}$ P. Gori, ${ }^{4,}{ }^{\ddagger}$ F. Bechstedt,${ }^{5,} \S$ D. S. Martin,${ }^{6,}$ ฯ \\ E. E. Barritt, ${ }^{6}$ A. Curcella, ${ }^{7}$ G. Prevot, ${ }^{7}$ and Y. Borensztein ${ }^{7, * *}$ \\ ${ }^{1}$ Istituto di Struttura della Materia-CNR (ISM-CNR), \\ Via Fosso del Cavaliere 100, 00133 Rome, Italy \\ ${ }^{2}$ Dipartimento di Fisica, Università di Roma "Tor Vergata", \\ Via della Ricerca Scientifica 1, 00133 Rome, Italy \\ ${ }^{3}$ INFN, Sezione di Roma Tor Vergata, Via della Ricerca Scientifica 1, 00133 Rome, Italy \\ ${ }^{4}$ Dipartimento di Ingegneria, Università Roma Tre, Via della Vasca Navale 79, 00146 Rome, Italy \\ ${ }^{5}$ IFTO, Friedrich Schiller Universität, Max-Wien Platz 1, 07743 Jena, Germany \\ ${ }^{6}$ Department of Physics, University of Liverpool L69 7ZE, UK \\ ${ }^{7}$ Sorbonne Université, CNRS-UMR 7588, Institut des NanoSciences de Paris, F-75005, Paris, France
}

\begin{abstract}
We present a state-of-the-art study of the optical properties of free-standing silicene and of singlelayer Si 1D and 2D nanostructures supported on $\operatorname{Ag}(110)$ and $\operatorname{Ag}(111)$ substrates. Ab initio simulations of reflectance anisotropy spectroscopy (RAS) and surface differential reflectivity spectroscopy (SDRS) applied to the clean Ag surface and $\mathrm{Si} / \mathrm{Ag}$ interfaces are compared with new measurements. For $\mathrm{Si} / \mathrm{Ag}(110)$ we confirm a pentagonal nanoribbon geometry, strongly bonded to the substrate, and rule out competing zigzag chain and silicenelike models. For $\mathrm{Si} / \mathrm{Ag}(111)$ we reproduce the main experimental features and isolate the optical signal of the epitaxial silicene overlayer. The absorption spectrum of a silicene sheet computed including excitonic and local field effects is found to be quite similar to that calculated within an independent particle approximation, and shows strong modifications when adsorbed on a $\mathrm{Ag}$ substrate. Important details of the computational approach are examined and the origins of the RAS and SDRS signals are explained in terms of the interface and substrate response functions. Our study does not find any evidence for Si adlayers that retain the properties of freestanding silicene.
\end{abstract}

\section{INTRODUCTION}

Silicene, the silicon-based counterpart of graphene represents an exciting new material merging the exceptional physical properties of graphene with the simplicity of integrating it in the already existing and largely developed silicon-based technology. ${ }^{1-5}$ A field-effect transistor has been reported at room temperature. ${ }^{6}$ However, there is also interest in silicene from a basic point of view because it is predicted to be a topological insulator which realises the quantum spin Hall phase effect. ${ }^{7}$

Freestanding ideal silicene presents a buckled honeycomb structure (Fig. 1(a)). Like graphene, silicene possesses (in the absence of spin-orbit corrections) massless fermions at the $\mathrm{K}$ point of the Brillouin zone. When spinorbit interaction is taken into account a gap as small as $1.5 \mathrm{meV}$ is created. ${ }^{8}$ Silicon (in contrast to carbon) is not able to create pure $\mathrm{sp}^{2}$ hybridization, hence the Si-Si bonds are somewhat intermediate between $\mathrm{sp}^{3}$ and $\mathrm{sp}^{2}$. This means that silicon atoms in silicene are quite reactive. When silicene is deposited on a substrate, interactions between silicon and substrate atoms cause deviations from the ideal geometry of freestanding silicene. Experimental studies and $a b$ initio density functional theory (DFT) calculations have shown that 1 monolayer (ML) of silicon atoms on a $\mathrm{Ag}(111)$ surface arranges in an apparently honeycomb structure (see Fig. 1(c)) but with a slight biaxial strain and $3 \times 3$ periodicity with respect to silicene. ${ }^{9-12}$ It was found to be thermodynamically stable across a wide range of chemical potential in Ref. 11, and recently confirmed experimentally using x-ray diffraction. ${ }^{13}$.

Besides the famous $(3 \times 3) /(4 \times 4)$ phase epitaxially grown on top of $\operatorname{Ag}(111),{ }^{9}$ other overlayer structures with $\sqrt{13} \times \sqrt{13}, \sqrt{7} \times \sqrt{7}$, and $2 \sqrt{3} \times 2 \sqrt{3}$ siliceneinduced reconstructions of the $\operatorname{Ag}(111)$ surface have been realised by depositing almost $1 \mathrm{ML}$ of silicon onto the silver substrate. ${ }^{14-16}$ Their atomic geometries have been explored by different techniques (see collections in Refs. 1 and 11). There are also several studies of the occupied electronic states of silicene on different substrates using angle-resolved photoemission spectroscopy (ARPES). $1,9,17,18$

Silicon deposition on $\mathrm{Ag}(110)$ instead gives rise to regular, ordered, submonolayer quantum wires. ${ }^{19}$ Based on scanning tunneling microscopy (STM) and ARPES experiments it was suggested that these (flat) wires could be nanoribbons (NRs) of silicene. ${ }^{20-22}$ Further studies combining STM, Si coverage measurements and DFT calculations ruled out all honeycomb-shaped models, however. ${ }^{23}$ Instead, a pentamer chain model (Fig. 1(d)) was proposed for the nanoribbon structures ${ }^{24}$ that satisfies DFT total energy calculations and high-resolution STM, core-level photoemission, and grazing-incidence $\mathrm{x}$ ray diffraction experiments. ${ }^{24,25}$ Further confirmation of a pentamer chain structure has recently been provided by photoelectron spectroscopy (XPS) and angle-resolved photoelectron diffraction (XPD) experiments, ${ }^{26}$ as well as non-contact atomic force microscopy (nc-AFM) and tip-enhanced Raman spectroscopy (TERS). ${ }^{27}$ Similar to $\mathrm{Si} / \mathrm{Ag}(111)$, the $\mathrm{Si} / \mathrm{Ag}(110)$ system thus consists of silicon adlayers strongly bonded to the Ag surface; nonethe- 
less there is little evidence to suggest that these structures retain any of the electronic features of a silicene-like layer, even when peeled off the substrate. ${ }^{24}$

Less effort has been devoted to understanding the optical signatures of silicenelike phases, their general sensitivity to optical probes, and the role the substrate plays in the optical response. Cinquanta et al. ${ }^{28}$ investigated the $\mathrm{Si} / \mathrm{Ag}(111)$ surface using transient-reflectance optical spectroscopy and performed DFT calculations of the dielectric functions for various $\mathrm{Si} / \mathrm{Ag}(111)$ phases, deducing a strong $\mathrm{Si}-\mathrm{Ag}$ hybridization and a surprising lack of sensitivity to the interface geometry. Deeper analysis can be provided by surface-sensitive spectroscopies like reflectance anisotropy spectroscopy (RAS) ${ }^{29}$ and surface differential reflectivity spectroscopy (SDRS). ${ }^{30}$ RAS has been successfully applied to the study of quasi-1D metal-semiconductor interfaces ${ }^{31,32}$, and should thus be appropriate for investigating the geometry of the highly anisotropic $\mathrm{Si} / \mathrm{Ag}(110)$ nanoribbon system. Previously, SDRS has been measured by some of us on monolayer $\mathrm{Si} / \operatorname{Ag}(110)^{33}$ and multilayer $\mathrm{Si} / \operatorname{Ag}(111)^{34,35}$. Comparison with $a b$ initio calculations of freestanding silicene ${ }^{36,37}$ found, in both cases, no evidence for silicenelike structures. Freestanding silicene is reported to exhibit a $\pi-\pi$ interband peak located near $2 \mathrm{eV}$ and an additional peak around 4 to $5 \mathrm{eV}$ due to a $\sigma-\sigma$ transition at the $\Gamma$ point. A joint theoretical-experimental study of surface optical properties should therefore be a key approach for determining whether silicon layers grown on a silver substrate do in fact possess electronic properties identical or close to those expected for freestanding silicene, as well to differentiate between similar structural geometries.

The paper is structured as follows. In Section II we summarize the theoretical and experimental methodologies. Section III reports our calculated optical spectra for bulk and surface Ag, highlighting some of the problems to be addressed in calculations of this nature. In Sections IV and V we present and discuss experimental data measured on silver-supported silicene-like systems, namely Si nanoribbons on $\mathrm{Ag}(110)$ and 2D honeycomb sheets on $\mathrm{Ag}(111)$, respectively. These data are compared with $a b$ initio calculations based on previously proposed structural models. Finally, in Section VI we discuss the optical spectra of freestanding silicene, in particular investigating the importance of many body effects on the optical response. The paper concludes with a summary and outlook.

\section{METHODOLOGY}

\section{A. Surface spectroscopy}

RAS and SDRS are two experimental techniques that probe the surface contribution to the reflectivity. RAS measures the difference in reflectivity $R$ for two perpendicular polarizations $x$ and $y$ of light in the plane of the surface. The isotropic contribution of the Ag substrate

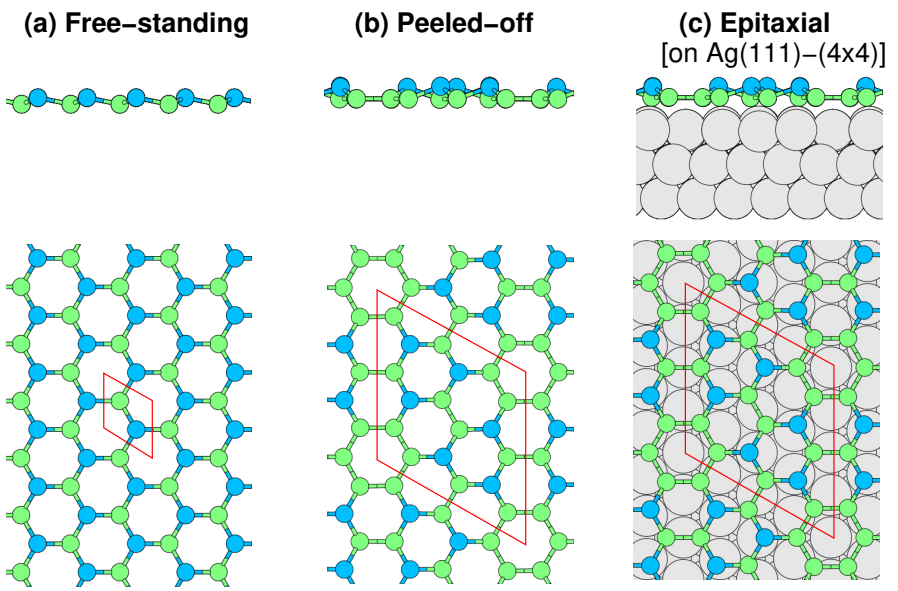

(d) Nanoribbons [on $\mathrm{Ag}(110)-(3 \times 2)$ and $\mathrm{Ag}(110)-(5 \times 2)$ ]

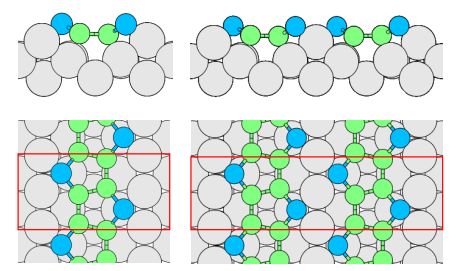

Pentamer chains (single \& double)
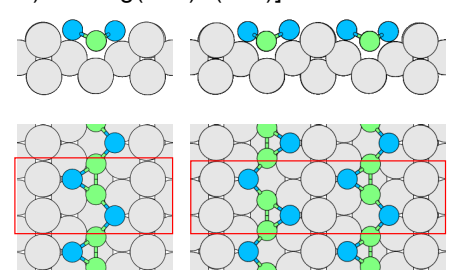

Zigzag chains (single \& double)
FIG. 1. Geometries of (a) freestanding silicene and (b) silicene peeled off the $\operatorname{Ag}(111)$ substrate, where it is grown as (c) epitaxial silicene. (d) $\mathrm{Si} / \mathrm{Ag}(110)$ single and double nanoribbons: pentamer chain and zigzag chain models. Blue and green balls represent up and down atoms of buckled silicon; grey is silver.

(bulk) cancels out, leaving a signal that is sensitive to anisotropic surface transitions:

$$
\left[\frac{\Delta R}{R}\right]_{\mathrm{RAS}}=\frac{R_{\mathrm{Si} / \mathrm{Ag}}^{x}-R_{\mathrm{Si} / \mathrm{Ag}}^{y}}{R} .
$$

In the case of a $\operatorname{Ag}(110)$ substrate, we choose $x=[1 \overline{1} 0]$ and $y=[001]$. Experimentally, the RAS signal is deduced from the relative variation of the complex reflection amplitude $\tilde{r}$ via $\Delta R / R \approx 2 \operatorname{Re}(\Delta \tilde{r} / \tilde{r})$, where $R=|\tilde{r}|^{2}$.

SDRS instead measures the difference in reflectivity between a clean surface and the same surface covered by an adsorbate layer. It is thus sensitive to all surface optical transitions (i.e., it can be used to probe $\mathrm{Si} / \mathrm{Ag}(111)$, whose RAS signal is zero by symmetry); furthermore, it can be performed with polarized or unpolarized light. Thus, for unpolarized light on a $\mathrm{Si} / \mathrm{Ag}$ interface $^{38}$ :

$$
\left[\frac{\Delta R}{R}\right]_{\mathrm{SDRS}}=\frac{R_{\mathrm{Si} / \mathrm{Ag}}-R_{\mathrm{Ag}}}{R_{\mathrm{Ag}}} .
$$

Generalization of this formula for polarized light is straightforward. In order to simplify the analysis of measured and computed RAS and SDRS spectra we make use of a three-layer model. ${ }^{39,40}$ In this way, the dielectric response of the semi-infinite system is approximated 
by (1) a layer of bulk (here, bulk Ag) with complex dielectric function $\tilde{\varepsilon}_{b}=\varepsilon_{b}^{\prime}+i \varepsilon_{b}^{\prime \prime} ;(2)$ an anisotropic surface layer of thickness $d$ with dielectric tensor $\tilde{\varepsilon}_{s}^{\alpha \beta}$ (where the subscript indicates a $\mathrm{Si} / \mathrm{Ag}$ interface or the clean $\mathrm{Ag}$ surface); and (3) a layer of vacuum. Frequency dependence of all $\tilde{\varepsilon}$ terms is understood throughout. We make the approximation that $d$ is unchanged with addition of Si. This allows us to decompose the SDRS and RAS into the real and imaginary parts of pure bulk and pure surface terms: ${ }^{41}$

$$
\begin{aligned}
& {\left[\frac{\Delta R}{R}\right]_{\mathrm{RAS}}=\frac{4 \omega d}{c}\left[A \cdot \Delta \varepsilon_{\mathrm{SDA}}^{\prime \prime}-B \cdot \Delta \varepsilon_{\mathrm{SDA}}^{\prime}\right],} \\
& {\left[\frac{\Delta R}{R}\right]_{\mathrm{SDRS}}=\frac{4 \omega d}{c}\left[A \cdot \Delta \varepsilon_{\mathrm{SDD}}^{\prime \prime}-B \cdot \Delta \varepsilon_{\mathrm{SDD}}^{\prime}\right]}
\end{aligned}
$$

where

$$
\begin{aligned}
A=\operatorname{Re}\left[\frac{1}{\tilde{\varepsilon}_{b}-1}\right] & =\frac{\varepsilon_{b}^{\prime}-1}{\left(1-\varepsilon_{b}^{\prime}\right)^{2}+\left(\varepsilon_{b}^{\prime \prime}\right)^{2}}, \\
B=-\operatorname{Im}\left[\frac{1}{\tilde{\varepsilon}_{b}-1}\right] & =\frac{\varepsilon_{b}^{\prime \prime}}{\left(1-\varepsilon_{b}^{\prime}\right)^{2}+\left(\varepsilon_{b}^{\prime \prime}\right)^{2}}
\end{aligned}
$$

Here we have defined two complex quantities, the surface dielectric anisotropy (SDA) appearing in the RAS:

$$
\Delta \tilde{\varepsilon}_{\mathrm{SDA}}=\tilde{\varepsilon}_{\mathrm{Si} / \mathrm{Ag}}^{x x}-\tilde{\varepsilon}_{\mathrm{Si} / \mathrm{Ag}}^{y y},
$$

where the subscripts indicate the $\mathrm{Si} / \mathrm{Ag}$ surface (a similar expression applies to the clean Ag surface), and an analogous term from SDRS that we call the surface dielectric difference (SDD):

$$
\Delta \tilde{\varepsilon}_{\mathrm{SDD}}=\tilde{\varepsilon}_{\mathrm{Si} / \mathrm{Ag}}-\tilde{\varepsilon}_{\mathrm{Ag}, \mathrm{s}},
$$

the last term indicating the clean Ag surface. Possible polarization dependence of the SDD is understood.

\section{B. Experiment}

Clean $\mathrm{Ag}(110)$ surfaces were prepared in UHV by repeated cycles of sputtering and annealing. Experiments were performed in an ultrahigh vacuum (UHV) environment with a base pressure in the $10^{-10}$ mbar region. Xray photoelectron spectroscopy (XPS) was used to confirm the absence of contaminants and low energy electron diffraction (LEED) was used to monitor atomic order. Si was deposited via an Omicron Focus EFM3 evaporator with the Ag crystal at room temperature, and coverage was determined by a combined analysis of XPS and LEED data. This preparation process results in growth of $0.8 \mathrm{~nm}$ wide single nanoribbons (SNRs), as confirmed by STM measurements, and is in accordance with previous STM/LEED analysis. ${ }^{42}$

Optical access into the UHV chamber was via a lowstrain window. RAS experiments were performed on two different $\operatorname{Ag}(110)$ crystals and during Si deposition the same characteristic spectral changes were observed from
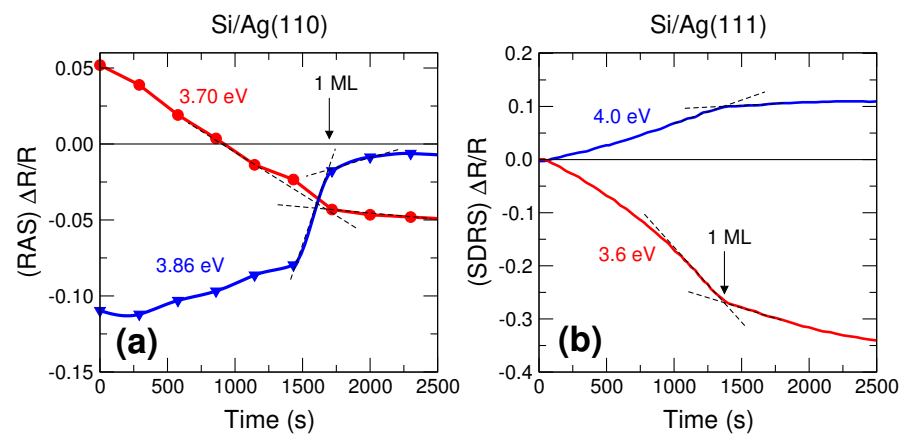

FIG. 2. Variation of the optical signal for two photon energies as a function of evaporation time: (a) RAS spectra of $\mathrm{Si} / \mathrm{Ag}(110)$ and (b) SDRS spectra of Si/Ag(111). Dashed lines indicate full completion of the Si overlayer.
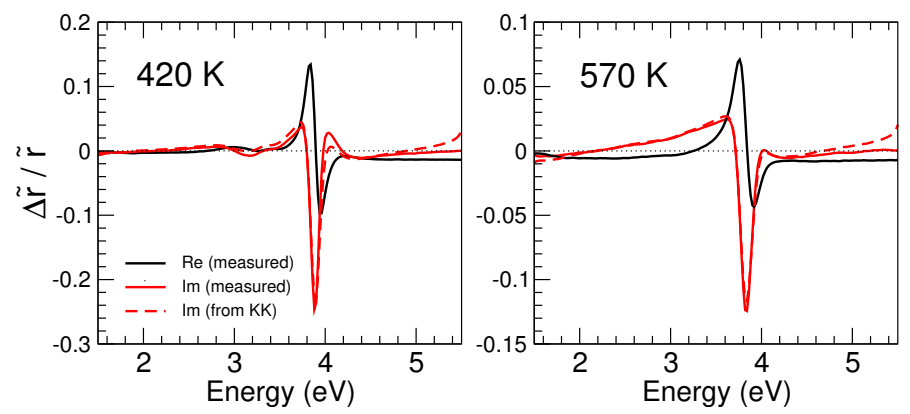

FIG. 3. Experimental RAS of $\mathrm{Ag}(110)$ in air at two temperatures. Plotted are the real and imaginary parts of $\Delta \tilde{r} / \tilde{r}$ (measurements) as well as the imaginary part computed using a Kramers-Kronig transform of the measured real part.

each crystal. Completion of the first Si ML was confirmed by the changes of slope of the signal recorded at two photon energies as a function of evaporation time, as shown in Fig. 2(a). Optical measurements for double nanoribbons (DNRs), obtained at higher temperature, have been reported elsewhere and the experimental details are given therein. ${ }^{33}$

The SDA for $\operatorname{Ag}(110)$ and $\mathrm{Si} / \operatorname{Ag}(110)$ were obtained by combining the real (Re) and imaginary ( Im) parts of the measured $\Delta \tilde{r} / \tilde{r}$ signal with $A$ and $B$ computed using standard dielectric function data ${ }^{43}$ of bulk $\mathrm{Ag}$, and then solving Eq. 3 for $\Delta R / R \approx 2 \operatorname{Re}(\Delta \tilde{r} / \tilde{r})$ and the corresponding equation for $\operatorname{Im}(\Delta \tilde{r} / \tilde{r})$. As $\operatorname{Im}(\Delta \tilde{r} / \tilde{r})$ is difficult to measure directly, Kramers-Kronig (KK) transforms of the measured $\operatorname{Re}(\Delta \tilde{r} / \tilde{r})$ signal were performed. The limited spectral range of the RAS data (generally 1.5 to 5.5 $\mathrm{eV}$ ) appears to be sufficient to generate an accurate $\mathrm{KK}$ transform for $\operatorname{Ag}(110)$. This is demonstrated in Fig. 3 where the experimentally measured Re and Im parts of the RAS response of an $\mathrm{Ag}(110)$ crystal in air are plotted (solid lines) for two sample temperatures (surface contamination is reduced at 570K). The measured Im part is compared to that calculated (dashed line) from a KK transform of the measured Re part. Here we use RAS data measured on a $\mathrm{Ag}(110)$ crystal in air ${ }^{44}$ in order to avoid first-order strain effects on the measured Im part 
that occur under UHV conditions due to the optical access window. ${ }^{29}$ As shown in Fig. 3, the shape of the calculated Im profile is in good agreement with the measured Im profile. We thus performed KK transforms on UHVmeasured (Re) RAS data on $\mathrm{Ag}(110)$ and $\mathrm{Si} / \mathrm{Ag}(110)$ (specifically, to obtain the data in Fig. 10).

Deposition of $\mathrm{Si}$ on $\mathrm{Ag}(111)$ was performed at $515 \mathrm{~K}$ and $600 \mathrm{~K}$. The quality of the surface and of the prepared phases were checked with LEED and with Auger electron spectroscopy. By comparing with previous LEED studies, ${ }^{45}$ we estimate that the $515 \mathrm{~K}$ phase is composed of about $30 \%(4 \times 4)$ phase and $70 \%(\sqrt{13} \times \sqrt{13})$ phase, while the $600 \mathrm{~K}$ surface is a majority of $(2 \sqrt{3} \times 2 \sqrt{3}) R 30^{\circ}$. As above, completion of the first Si ML was determined by the changes of slope of SDRS transients, shown in Fig. 2(b) for the case of $\mathrm{Si} / \mathrm{Ag}(111)$ grown at $515 \mathrm{~K}$. The SDD were described by a sum of several Lorentzian functions for $\mathrm{Si} / \mathrm{Ag}(111)$, therefore exactly fulfilling the Kramers-Kronig relations, and their parameters were obtained by fitting the experimental SDR spectra using Eq. 4. A complete description of the procedure is given in Ref. 33 for the detailed investigation of the $\mathrm{Si} / \mathrm{Ag}(110)$ system. In this latter case, only one Lorentzian function was enough to reproduce the experiment.

\section{Ab initio theory}

Calculations were carried out using DFT in the local density approximation (LDA). ${ }^{46,47}$ We use a planewave/norm-conserving pseudopotential framework as implemented in the quantum-ESPRESSO code. ${ }^{48} \mathrm{Pe}-$ riodically repeated supercells containing symmetric slabs, or 2D layers, separated by vacuum regions were used to simulate the $\mathrm{Si} / \mathrm{Ag}$ surfaces and silicene layers. We used a kinetic energy cutoff of $40 \mathrm{Ry}$ and the LDA lattice constant of $a_{0}=4.075 \AA$. For $\mathrm{Si} / \operatorname{Ag}(111)$ we used a symmetric 7 layer slab of $4 \times 4$ periodicity (about $20 \AA$ thick). For $\mathrm{Si} / \mathrm{Ag}(110), 3 \times 2(\mathrm{SNRs})$ and $5 \times 2(\mathrm{DNRs})$ supercells were used containing slabs of 13 atomic layers (about $19 \AA$ thick).

Optical properties were then calculated at different levels of theory (and corresponding computational effort) for different systems. For the $\mathrm{Si} / \mathrm{Ag}$ slabs we use the simplest independent particle (IP) approach (equivalently, the RPA level without local field effects) based on the DFT eigenvalues, as implemented in the Yambo code ${ }^{49}$. Within DFT-IP, the imaginary part of the supercell (SC) dielectric function (i.e. slab plus vacuum region) is computed as:

$$
\begin{aligned}
\operatorname{Im}\left[\varepsilon_{s}^{\mathrm{SC}, \alpha \alpha}\right]=\frac{8 \pi e^{2}}{m^{2} \omega^{2} A L} \sum_{\mathbf{k}} & \sum_{v, c}\left|\mathbf{p}_{v c}^{\alpha}(\mathbf{k})+i\left[V^{\mathrm{NL}}, \mathbf{r}^{\alpha}\right]\right|^{2} \\
& \times \delta\left(E_{c}(\mathbf{k})-E_{v}(\mathbf{k})-\hbar \omega(8)\right.
\end{aligned}
$$

where $A$ and $L$ denote the cell area and height, respectively, and $E_{c, v}(\mathbf{k})$ are the DFT Kohn-Sham (KS) single particle eigenvalues. Dense k-point meshes of $12 \times 12 \times 1$ for the $\mathrm{Si} / \mathrm{Ag}(111)(4 \times 4)$ cell and $16 \times 32 \times 1$ for the $\mathrm{Si} / \operatorname{Ag}(110)(3 \times 2)$ cell were found to yield well converged spectra. The transition matrix elements contain the momentum operator $\mathbf{p}$ and a commutator term deriving from the nonlocal components of the pseudopotentials, $V^{\mathrm{NL}} \cdot{ }^{50}$ For $\mathrm{Ag}$, the $V^{\mathrm{NL}}$ term can imply a heavy computational penalty, especially for slabs containing many atoms, and is thus often omitted in $a b i n i$ tio calculations. ${ }^{28}$ The same formula holds for bulk calculations, with $A \cdot L$ replaced by the volume $V$ of the primitive cell, and was used to calculate $\varepsilon_{b}^{\prime \prime}$ for bulk Ag. Surface dielectric functions $\varepsilon_{s}$ can be derived from the supercell $\varepsilon_{s}^{\mathrm{SC}}$ by appropriately accounting for the presence of two equivalent surfaces and renormalizing to $d$. It holds that: ${ }^{51}$

$$
\begin{aligned}
d \Delta \tilde{\varepsilon}_{\mathrm{SDA}} & =d\left(\tilde{\varepsilon}_{\mathrm{Si} / \mathrm{Ag}}^{x x}-\tilde{\varepsilon}_{\mathrm{Si} / \mathrm{Ag}}^{y y}\right) \\
& =\frac{L}{2}\left(\tilde{\varepsilon}_{\mathrm{Si} / \mathrm{Ag}}^{\mathrm{SC}, x x}-\tilde{\varepsilon}_{\mathrm{Si} / \mathrm{Ag}}^{\mathrm{SC}, y}\right), \\
d \Delta \tilde{\varepsilon}_{\mathrm{SDD}} & =d\left(\tilde{\varepsilon}_{\mathrm{Si} / \mathrm{Ag}}-\tilde{\varepsilon}_{\mathrm{Ag}, \mathrm{s}}\right) \\
& =\frac{L}{2}\left(\tilde{\varepsilon}_{\mathrm{Si} / \mathrm{Ag}}^{\mathrm{SC}}-\tilde{\varepsilon}_{\mathrm{Ag}, \mathrm{s}}^{\mathrm{SC}}\right) .
\end{aligned}
$$

Since the surface layer thickness $d$ is not well defined, only quantities like $d \tilde{\varepsilon}$ or $d \Delta \tilde{\varepsilon}$ are accessible from Eqs. 9 and 10 , and $d \Delta \tilde{\varepsilon}$ can be compared with the experimental one.

The DFT-IP scheme has been widely used to compute surface optical spectra of semiconductors, especially when used in conjunction with a simple "scissors" shift to correct the (underestimated) DFT-LDA eigenvalues. This scheme is not easily applicable to metallic systems. Furthermore, many-body effects may be especially important in low dimensional systems. For this reason we calculate the electronic and optical properties of ideal freestanding silicene including many-body effects by following a standard three-step procedure. The first step consists of a DFT calculation of the KS eigenstates and eigenvalues, as before. Secondly, independent quasiparticle (IQP) corrections to the KS states are computed within the $G W$ approximation, ${ }^{52}$ where $G$ is the single particle Green's function and $W$ the screened Coulomb interaction. In the final step, the Bethe-Salpeter equation for the optical response is solved which accounts for excitonic and local field effects. ${ }^{52}$

The GW calculations were performed in the usual oneshot $\left(G_{0} W_{0}\right)$ approach, applying a $50 \times 50 \times 1(90 \times 90 \times$ $1)$ k-point mesh for the screened (exchange) part of the self-energy and the plasmon-pole approximation for the screening dynamics. 300 bands have been used for the screening. The Coulomb interaction between the sheets of the superlattice was cut. For the BSE calculation, $50 \times 50 \times 1 \mathrm{k}$-points, four filled valence and five empty conduction bands were included. The dp4exc code was used $^{53}$. 


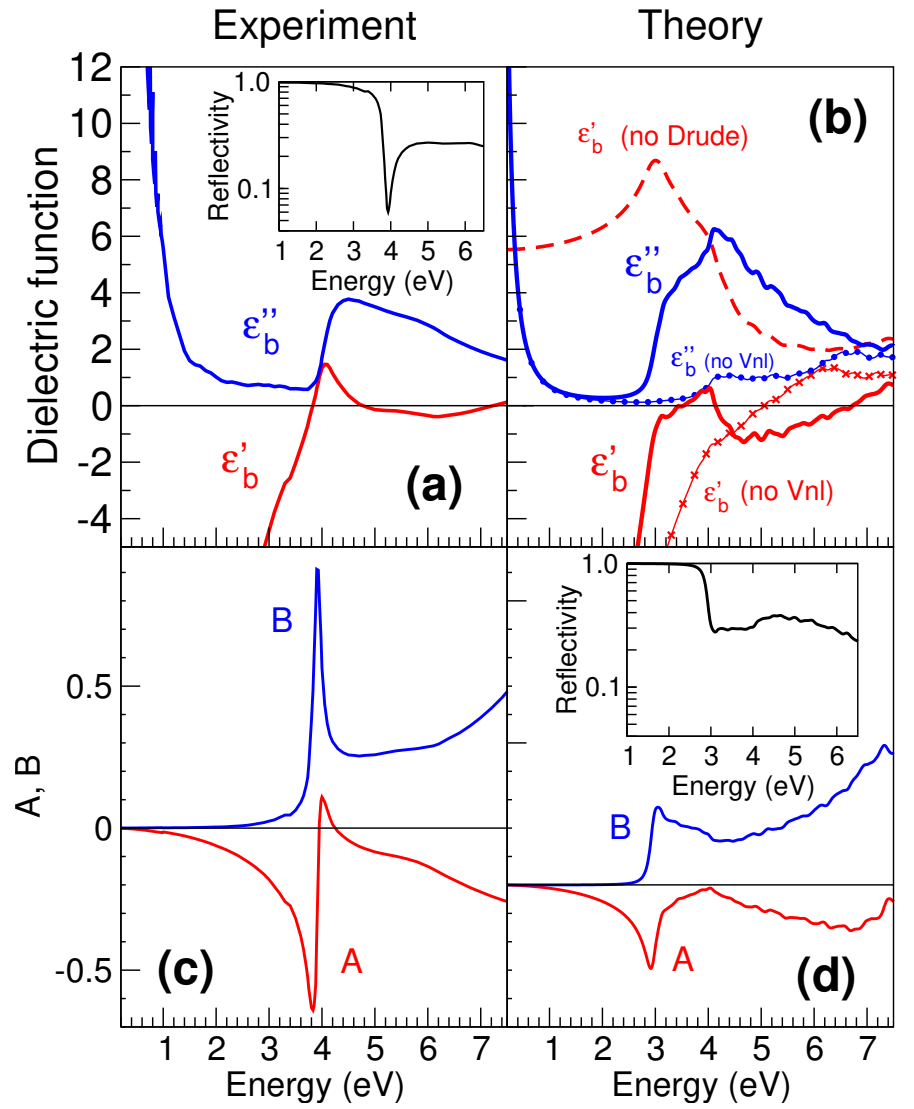

FIG. 4. Dielectric function of bulk Ag. Left panels: experiment, right panels: theory. (a) Data from Palik. ${ }^{54}$ (b) Full calculations of $\varepsilon_{b}^{\prime}, \varepsilon_{b}^{\prime \prime}$. Also shown: $\varepsilon_{b}^{\prime}$ without the Drude term (dashed line); $\varepsilon_{b}^{\prime}, \varepsilon_{b}^{\prime \prime}$ without the $V^{\mathrm{NL}}$ term (dotted lines). Panels (c) and (d): $A$ and $B$ terms. Insets: reflectivity.

\section{AB INITIO CALCULATIONS OF SILVER}

Equations 3-5 show that the RAS and SDRS depend on a delicate interplay between the optical properties of the surface/adlayer and that of the substrate. It has been noted elsewhere ${ }^{40}$ that $\mathrm{Ag}$ systems present a particularly complicated case due to the forms of $A$ and $B$, and thus analysis and computation of RAS and SDRS require care. In the following we examine in turn the optical response of bulk and surface Ag.

\section{A. Bulk Ag}

Experimental data ${ }^{54}$ for $\tilde{\varepsilon}_{b}$ is shown in Fig. 4(a). The real part crosses zero close to $3.9 \mathrm{eV}$, which also marks the onset of interband transitions seen in the imaginary part. The reflectivity $R$ (inset) is sharply peaked at $3.9 \mathrm{eV}$. The $A$ and $B$ terms, shown in panel (c), are particularly sharp, in contrast to many other semiconductors and metals. ${ }^{40}$

Calculations of $\tilde{\varepsilon}_{b}, A, B$, and $R$ are shown in Fig. 4(b) and (d). To account for intraband contributions, we add
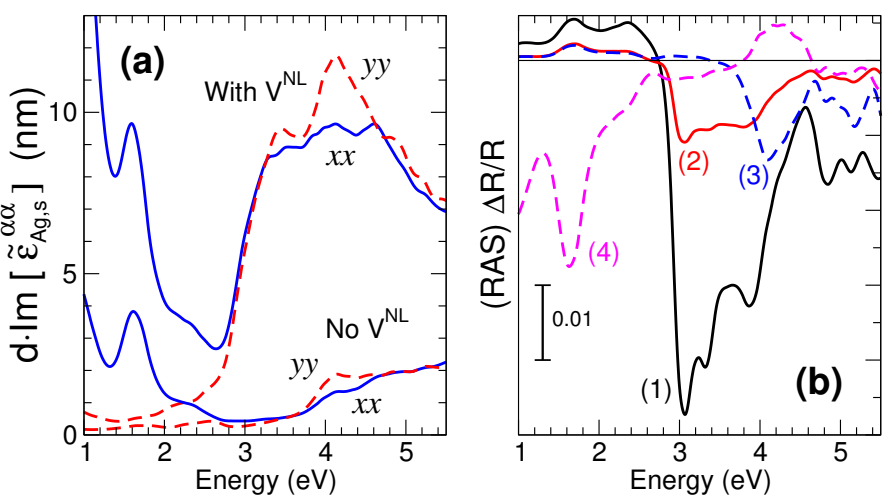

FIG. 5. (a) Clean $\operatorname{Ag}(110)$ surface dielectric function times $d$ with (upper) and without (lower) the nonlocal commutator term $V^{\mathrm{NL}}$ (Eq. 8). Solid blue lines: $x=[1 \overline{1} 0]$. Dashed red lines: $y=$ [001]. (b) Influence of $V^{\mathrm{NL}}$ and Drude term (Eq. 11) on the computed RAS. (1) Full calculation, includ$\operatorname{ing} V^{\mathrm{NL}}$ in $\tilde{\varepsilon}_{\mathrm{Ag}, \mathrm{s}}^{\alpha \alpha}$ and $\tilde{\varepsilon}_{b}$, and a Drude term in $\tilde{\varepsilon}_{b}$. (2) As (1), without $V^{\mathrm{NL}}$ in $\tilde{\varepsilon}_{\mathrm{Ag}, \mathrm{s}}^{\alpha \alpha}$. (3) As (1), without $V^{\mathrm{NL}}$ in $\tilde{\varepsilon}_{\mathrm{Ag}, \mathrm{s}}^{\alpha \alpha}$ and $\tilde{\varepsilon}_{b}$. (4) As (3), with no Drude term in $\tilde{\varepsilon}_{b}$.

a simple Drude term to the interband part represented by Eq. 8:

$$
\tilde{\varepsilon}_{D}(\omega)=-\frac{\omega_{D}^{2}}{\omega(\omega+i \eta)}
$$

where $\omega_{D}=9.48 \mathrm{eV}$ is the Drude plasma frequency ${ }^{55}$ and we set $\eta=0.133 \mathrm{eV} .{ }^{43}$

There are considerable discrepancies with respect to the experiment. Firstly, the computed $\varepsilon_{b}^{\prime \prime}$ is redshifted by about $1 \mathrm{eV}$, and overestimates the intensity. The onset of absorption thus occurs at about $3.0 \mathrm{eV}$, rather than near $3.9 \mathrm{eV}$ where the bulk plasma frequency occurs. The importance of the Drude term is demonstrated in the figure for $\varepsilon_{b}^{\prime}$. The decrease of reflectivity at $3 \mathrm{eV}$ is also less sharp and less deep than the experimental data at $3.9 \mathrm{eV}$. The inclusion of $V^{\mathrm{NL}}$ terms are shown to be critical in the case of bulk Ag, strongly modifying the intensity and lineshape of both $\varepsilon_{b}^{\prime}$ and $\varepsilon_{b}^{\prime \prime}$. Last, the $A$ and $B$ terms are 3-4 times weaker than the experimental ones, are redshifted, and lack sharpness. These results are nonetheless typical of what is possible within DFT-LDA. To obtain an improved agreement with experiment, quasiparticle corrections are needed, as demonstrated by $G W$ calculations of the silver reflectance spectrum in Ref. 55. Simple scissors-like corrections are not possible in this case. Hence, all spectra in the rest of this paper will necessarily appear redshifted with respect to experiment by about $0.9 \mathrm{eV}$. Moreover, the features corresponding to $A$ and $B$ will appear also less sharp in the calculated spectra with respect to the experimental ones.

\section{B. Ag surface}

The surface optical spectra of $\operatorname{Ag}(110)$ have been thoroughly studied in the past both experimentally ${ }^{56-58}$ 
and theoretically using dipolar models ${ }^{59}$ and $a b$ initio LMTO methods. ${ }^{60,61}$ Here we revisit the RAS calculation in order to demonstrate the level of agreement with experiment for the clean Ag surface expected using our planewave/pseudopotential approach, to demonstrate some important technical approximations, and to illustrate the spectral decomposition in terms of surface and bulk contributions. These technical details are equally relevant for $\mathrm{Ag}(111)$.

The in-plane components of the computed dielectric tensor of the $\operatorname{Ag}(110)$ slab is shown in Fig. 5(a). As in the bulk case, the $V^{\mathrm{NL}}$ term has a huge contribution to the slab $\varepsilon_{\mathrm{Ag}, \mathrm{S}}^{\alpha \alpha}$ and its inclusion is crucial for obtaining the correct amplitude and lineshape. The differences between the $x x$ and $y y$ components are clearly enhanced. Panel (b) shows the corresponding RAS spectra. Following Monachesi et al. ${ }^{61}$ we add a Drude term to the bulk $\varepsilon_{b}$ via Eq. 11 but not to the surface $\varepsilon_{\mathrm{Ag}, \mathrm{s}}^{\alpha \alpha}$ as it is expected to (mostly) cancel out in the SDA. The RAS spectrum obtained from a full calculation including both $V^{\mathrm{NL}}$ and the Drude component is indicated as (1) in the figure. It compares favourably with the spectrum reported by Monachesi et al, except for a small energetic shift.

Omitting the $V^{\mathrm{NL}}$ term in the SDA produces curve (2). As expected, the intensity is greatly reduced. However, the overall lineshape is not considerably different from the full spectrum (1). Thus, omission of $V^{\mathrm{NL}}$ from the SDA in computing the RAS may be a viable option, due to cancellations occurring in Eq. 3. On the other hand, omission of $V^{\mathrm{NL}}$ from the bulk $\varepsilon_{b}$ yields a qualitatively incorrect spectrum, as shown by (3). Last, curve (4) demonstrates that inclusion of a Drude term in $\varepsilon_{b}$ is crucial for the RAS, and as noted previously, ${ }^{61}$ even changes its sign. Clearly, the message here is that $\varepsilon_{b}$ must be com-
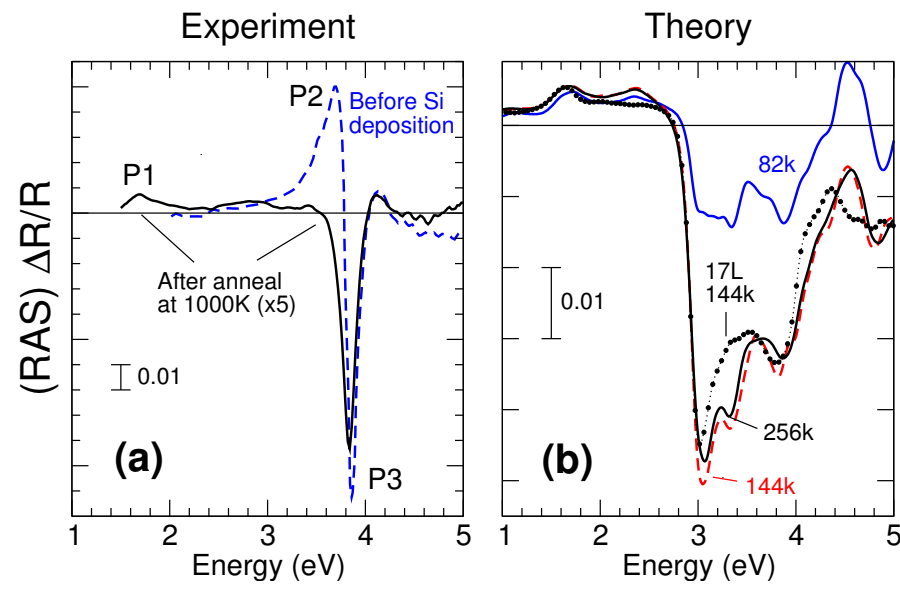

FIG. 6. RAS of clean $\operatorname{Ag}(110)$. (a) Experimental data on a high-quality surface annealed at $1000 \mathrm{~K}$ (solid black line, scaled $\times 5$ ) and on the present Ag surface before Si deposition (blue dashed line). (b) Calculations for a 13-layer slab using 82, 144, and $256 \mathrm{k}$-points, as well as for a 17-layer slab with 144 k-points (dotted). The horizontal line indicates the zero of the $Y$-axis in each case; note the difference in $Y$-scale between the two panels. puted to the best extent possible, and that $V^{\mathrm{NL}}$ has to be included, at least for the bulk, and preferentially also for the surface.

The computed RAS are now compared with experiment in Fig. 6. In (a), we report data for a 'pure' single terraced $\operatorname{Ag}(110)$ surface prepared by repeated annealing to $1000 \mathrm{~K}$, and on the standard prepared surface prior to Si deposition. Peak P1, visible in the former data, is a true surface-state transition. As discussed in Ref. 58, peak P2 is associated with sample roughness, and should thus be absent from our computed spectra. All reported spectra in the literature show a sharp peak P3 with magnitude ranging from 0.012 to $0.100,{ }^{57,58,62}$ lying close to the bulk plasma frequency of silver. Thus both P1 and P3 should be accessible to theory.

The calculations in panel (b) reveal that our RAS spectrum using $256 \mathrm{k}$-points is well converged. Using a thicker slab (17 layers) reveals that the features at $2.4 \mathrm{eV}$ and $3.4 \mathrm{eV}$ are spurious. Thus, we succeed in reproducing well the $\mathrm{P} 1$ peak (the optically active states lie close to the Fermi level, and thus experience weak quasiparticle corrections), as well as the main P3 peak. The latter is redshifted by $0.9 \mathrm{eV}$ for reasons discussed earlier, and the intensity is somewhat underestimated. There is also an incorrect feature near $3.8 \mathrm{eV}$. These discrepancies can be traced back to the poor description of the bulk reflectivity in Fig. 4.

\section{SILICON NANORIBBONS: Si/Ag(110)}

We now extend our study of the optical response of $\mathrm{Ag}(110)$ to the $\mathrm{Si} / \mathrm{Ag}(110)$ nanoribbon (NR) system. As noted in the introduction, several structural models have been proposed, with the best being the pentamer chain model shown in Fig. 1(d) (being the most thermodynamically stable, and in closest overall agreement with experiments). To illustrate optical sensitivity to local geometry, we also consider the zigzag chain model proposed in Ref. 23 and shown in Fig. 1(d). As the NRs constitute a quasi-1D system, we can use both RAS as well as polarization-dependent SDRS to analyse the surface.

Using the same convergence parameters as before for the clean $\operatorname{Ag}(110)$ surface, the SDRS (Fig.7) was computed for both models and for two light polarizations parallel to $(x)$ and perpendicular to $(y)$ the nanoribbon direction. These spectra are compared to previously published SDRS data ${ }^{33}$ for the double nanoribbon-covered surface. As in the case of clean $\operatorname{Ag}(110)$, the intraband contribution to the SDD is neglected.

The experimental data [Fig.7(a)] consists of a derivative-like resonance around the bulk plasma frequency of Ag, confirming the substrate origins of the SDRS signal. A slightly broader and larger amplitude profile is observed for the parallel component with respect to the perpendicular one. Our computed SDRS spectra (panels (b) and (c)) yield similar derivative-like lineshapes, with the usual redshift and reduced intensity. 


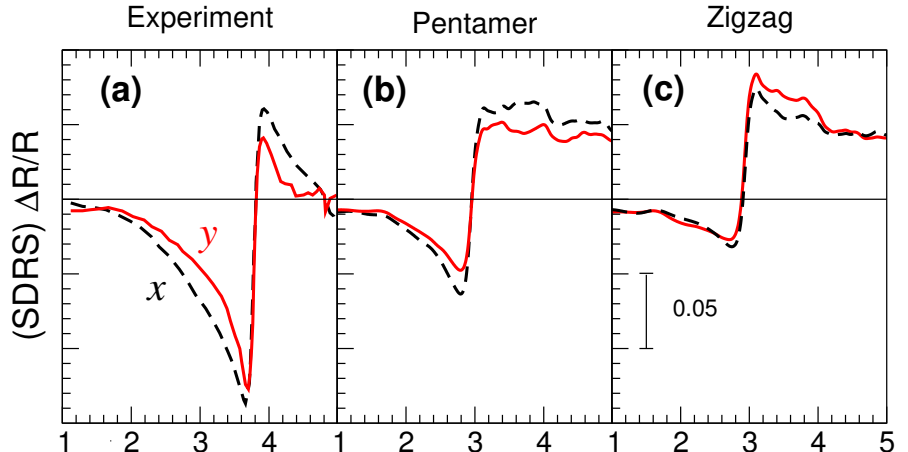

FIG. 7. SDRS of $\mathrm{Si} / \operatorname{Ag}(110)$ for polarization parallel $(x=$ [110], dashed black lines) and perpendicular $(y=[001]$, solid red lines) to the nanoribbons. (a) Experiment (DNRs, Ref. 33). (b) Theory: pentamer chain model (DNRs). (c) Zigzag model (SNRs). $X$ and $Y$ scales are the same in each panel.
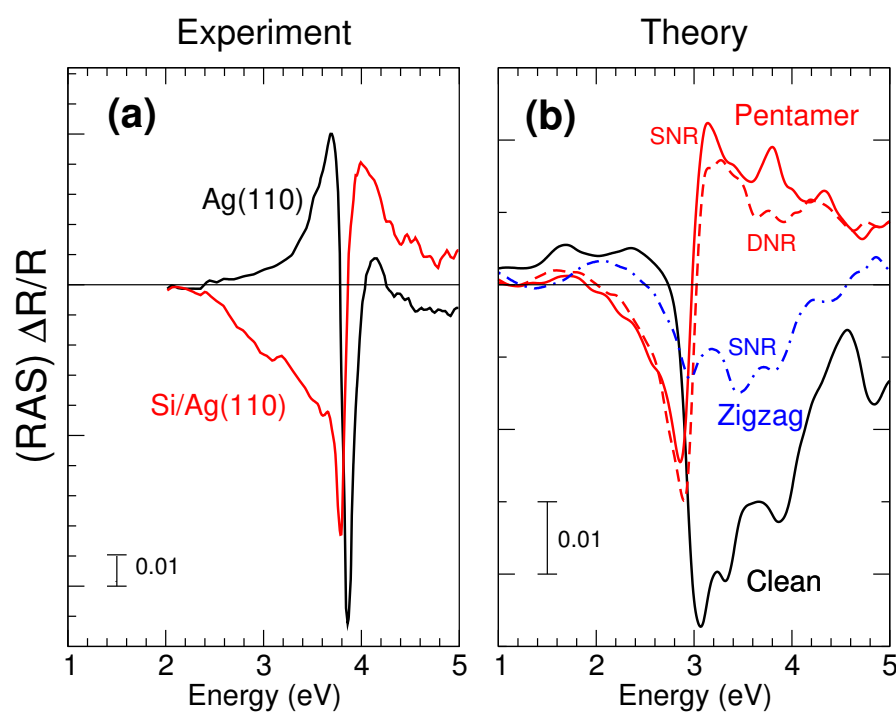

FIG. 8. RAS of Si/Ag(110). (a) Experimental data before and after deposition of 1 ML Si nanoribbons at RT. (b) Computed spectra for the clean surface, pentamer chain model (SNR and DNR) and zigzag chain model (SNR).

We can tentatively distinguish between the two models by considering the polarization dependence. The pentamer chain model, like the experiment, shows a lower intensity for the $y$ polarization across the full spectral range. The opposite occurs for the zigzag chain model.

This polarization dependence suggests that RAS should be a more useful technique for studying the $\mathrm{Si} / \mathrm{Ag}(110)$ system. Fig. 8(a) compares the measured RAS of the clean $\mathrm{Ag}(110)$ surface (standard preparation) with the signal obtained after Si deposition until full coverage formation of single nanoribbons. Si deposition induces strong changes in the RAS. The main negative peak is weakened and redshifted by $0.1 \mathrm{eV}$ and a broad negative signal appears below it in the 2.4 to $3.7 \mathrm{eV}$ range. Furthermore, the spectrum becomes positive above $4 \mathrm{eV}$, so that the overall signal has a derivativelike lineshape.

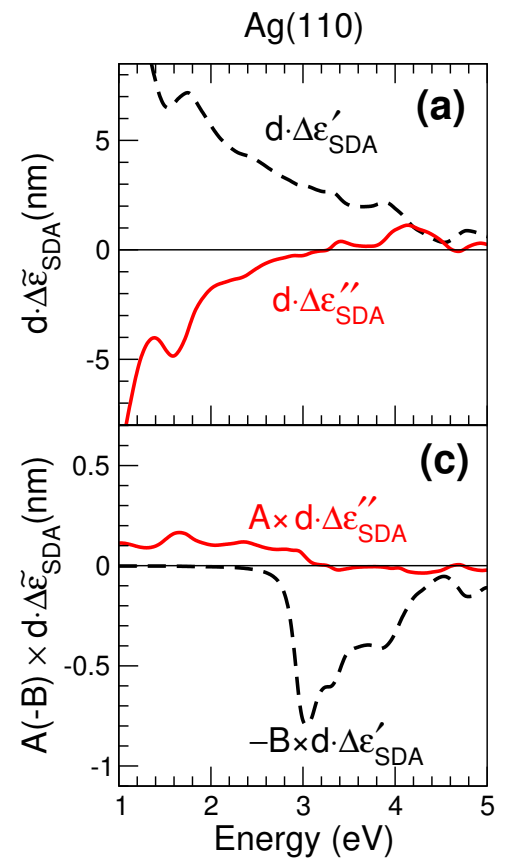

$\mathrm{Si} / \mathrm{Ag}(110) \mathrm{SNR}$

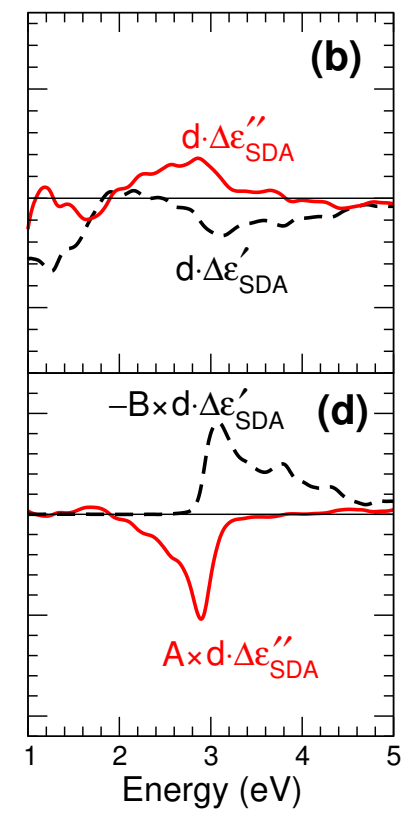

FIG. 9. Calculated SDA for clean $\mathrm{Ag}(110)$ and the $\mathrm{Si} / \mathrm{Ag}(110)$ single nanoribbon system. Panels (a) and (b): components of $d \Delta \tilde{\varepsilon}_{\mathrm{SDA}}$. Panels (c) and (d): as (a) and (b), but including the $A$ and $-B$ prefactors as they appear in Eq. 3. X- and Y-scales are the same for (a) and (b), and for (c) and (d), respectively.

RAS calculations are shown in panel (b) for the pentamer chain (SNR and DNR) and zigzag (SNR only) models. In contrast to the SDRS in Fig. 7, RAS clearly enhances the small structural differences between the two models. The pentamer model reproduces the main features of the experimental RAS. More precisely, it reproduces the redshift of about $0.1 \mathrm{eV}$ in the main negative peak and the broad negative tail below it. The change in sign above $3 \mathrm{eV}$ is also evident. These features are mostly missing from the zigzag model. It is also notable that the signal for SNR and DNR reconstructions are practically identical, at least below $3.5 \mathrm{eV}$. Moreover, the agreement with experiment for $\mathrm{Si} / \mathrm{Ag}(110)$ is overall better than for the clean $\mathrm{Ag}(110)$ surface thanks to the removal of the positive peak P2. As explained above, P2 arises from local roughness such as vacancies or step edge defects induced by the ion bombardment procedure used to clean the Ag surface. However, it has been shown that the formation of (double) Si nanoribbons is accompanied by an important reconstruction of the Ag surface whereby two of every five $\mathrm{Ag}$ rows are removed. The ejected $\mathrm{Ag}$ atoms diffuse on the surface to form new Ag terraces. ${ }^{63}$ Such reorganization of surface atoms could therefore "repair" the surface and remove the local defects induced by ion bombardment. The good agreement between the calculated and experimental RAS thus constitutes further evidence for the pentamer chain model of the $\mathrm{Si} / \mathrm{Ag}(110)$ interface. 

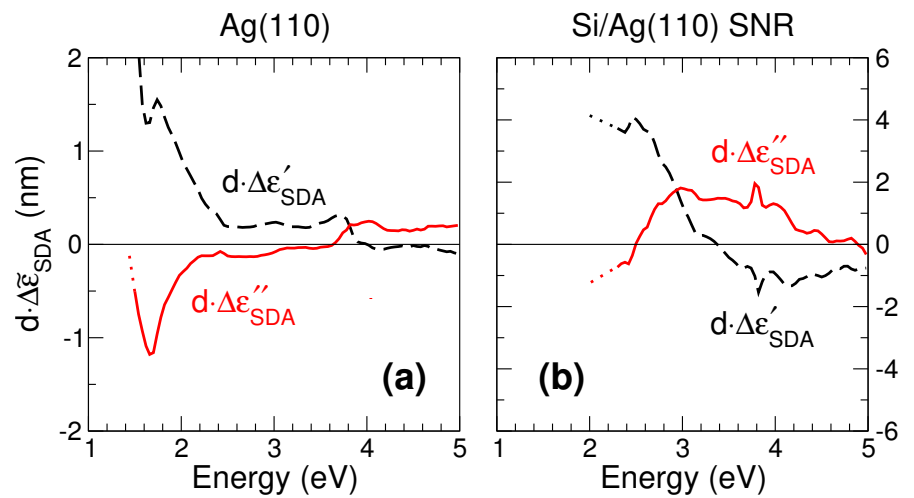

FIG. 10. Experimental SDA for (a) clean Ag(110) (corresponding to post-anneal data) and (b) with Si nanoribbons deposited at RT. Kramers-Kronig transforms have been used to extract these data (see Sec. IIB).

The changes observed in the RAS signal with addition of Si can be understood by examining the calculated SDA $\left(\Delta \tilde{\varepsilon}_{\mathrm{SDA}}\right)$ for the clean and Si-covered surfaces in conjunction with the $A$ and $B$ terms plotted in Fig. 4(d). The SDA, and the SDA multiplied by $A$ and $-B$, are shown in Fig. 9. Recall that the RAS is related to the sum of the latter quantities via Eq. 3 . The $\Delta \tilde{\varepsilon}_{\mathrm{SDA}}$ terms appear relatively broad and featureless in comparison to the sharper $A$ and $B$ terms. For the specific case of $\mathrm{Ag}$ substrates, the SDA can therefore be understood as a weighting factor of the $A$ and $-B$ terms in forming the composite RAS signal. In the clean surface, $\Delta \varepsilon_{\text {SDA }}^{\prime \prime}$ is almost zero around $3 \mathrm{eV}$. Thus the RAS (shown in Fig. 8(b)) is largely dominated by the sharp $B$ term, peaked at $3 \mathrm{eV}$, weighted by the positive $\Delta \varepsilon_{\text {SDA }}^{\prime}$. For the $\mathrm{Si} / \mathrm{Ag}(110)$ surface, both real and imaginary parts of the SDA contribute to the RAS around $3 \mathrm{eV}$ in equal amounts. In particular, the $A$ term now gives rise a negative component (solid line, panel (d)) with a peak at $2.9 \mathrm{eV}$ and a broad tail below this. This explains the small $0.1 \mathrm{eV}$ redshift observed in the RAS upon addition of $\mathrm{Si}$ as well as the appearance of the broad tail. The $\Delta \varepsilon_{\text {SDA }}^{\prime}$ term has an opposite sign to that of the clean surface: when multiplied by $-B$ it gives rise to a positive peak at $3 \mathrm{eV}$. Summing the two components thus gives rise to the derivativelike RAS signal of $\mathrm{Si} / \mathrm{Ag}(110)$.

Our analysis is supported by experimental extraction of the SDA from the measured RAS following the procedure outlined in Sec. IIB. Fig. 10 shows $\Delta \tilde{\varepsilon}_{\text {SDA }}$ for the clean surface annealed to $1000 \mathrm{~K}$ (corresponding to the RAS in Fig. 6(a), solid curve) and for the $1 \mathrm{ML}$ $\mathrm{Si} / \mathrm{Ag}(110)$ SNR interface. Data is available only within the range of the measured RAS signal $(1.5-5.0 \mathrm{eV})$. Furthermore, for $\mathrm{Si} / \mathrm{Ag}(110)$, both the RAS and $B$ terms are close to zero below about $2.4 \mathrm{eV}$, making inversion of Eq. 3 unreliable. Hence, the extracted SDA signal below $2.4 \mathrm{eV}$ has been omitted from Fig. 10(b). In spite of these restrictions, the experimental SDA show general good agreement with the theoretical data shown in Fig. 9(a) and (b). For the clean surface, $\Delta \varepsilon_{\mathrm{SDA}}^{\prime \prime}$ clearly shows a fea-
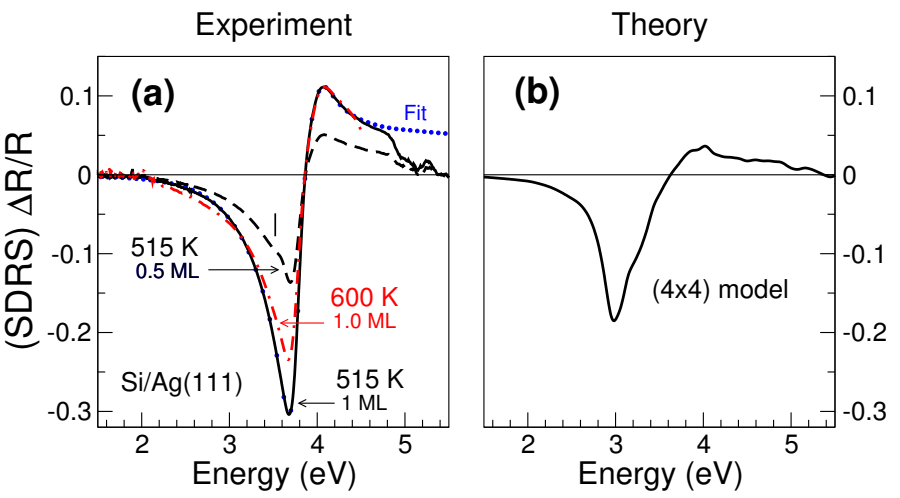

FIG. 11. SDRS of Si/Ag(111): (a) Experimental measurements at $515 \mathrm{~K}$ for $0.5 \mathrm{ML}$ (dashed black line) and 1.0 ML (solid black line ) and at $600 \mathrm{~K}$ for $1.0 \mathrm{ML}$ (dot-dashed red line). The Lorentzian fit to the $1.0 \mathrm{ML} 515 \mathrm{~K}$ data is superimposed (blue dots). (b) Computed spectrum for the $(4 \times 4)$ model (corresponding to 1.0 ML coverage).

ture at $1.7 \mathrm{eV}$ that explains the surface state peak $\mathrm{P} 1$ in the RAS, as previously noted. ${ }^{57}$ In the $\mathrm{Si} / \mathrm{Ag}(110)$ case, the two components contribute with the same magnitude around the experimental plasma frequency $(3.9 \mathrm{eV})$, in agreement with the theoretical prediction (theoretical plasma frequency equal to $3 \mathrm{eV}$ ). Moreover, taking into account the $0.9 \mathrm{eV}$ shift between experiment and theory, the crossing points of $\Delta \varepsilon_{\mathrm{SDA}}^{\prime}$ and $\Delta \varepsilon_{\mathrm{SDA}}^{\prime \prime}$ occur at consistent energies. On the other hand, the intensity at the crossing point differs between theory and experiment, and agreement diverges further at lower energies. There are several possible reasons for these discrepancies. On the theory side, this includes lack of consideration of intraband anisotropy in $\operatorname{Ag}(110)$, which can be modified upon formation of Si nanoribbons, as well as omission of many body effects on the lineshape. On the experimental side, the possible presence of anisotropic optical transitions at energies higher than the present energy range could give rise to a positive or negative constant contribution to $\Delta \varepsilon_{\mathrm{SDA}}^{\prime}$ when the KK transform is performed. In spite of these considerations, analysis of the SDA and $A$ and $B$ terms yields a consistent and almost thorough explanation of all features observed in the experimental and theoretical RAS spectra of clean and Si-covered $\operatorname{Ag}(110)$.

\section{EPITAXIAL SILICENE: $\mathrm{Ag}(111): \mathrm{Si}$}

We now move to the case of a single $2 \mathrm{D}$ silicon sheet adsorbed on $\operatorname{Ag}(111)$, which we refer to as "epitaxial silicene" after Vogt et al. ${ }^{9}$ SDRS spectra measured for a coverage of 1 ML of silicon are presented in Fig. 11(a) for two different growth temperatures. They are characterized by a single main oscillation around $3.8 \mathrm{eV}$ and are zero below $2 \mathrm{eV}$. A Lorentzian fit to the $1 \mathrm{ML}$ data, obtained using the procedure described in Sec. II B, matches the data perfectly below $5 \mathrm{eV}$. A half-monolayer spectrum 


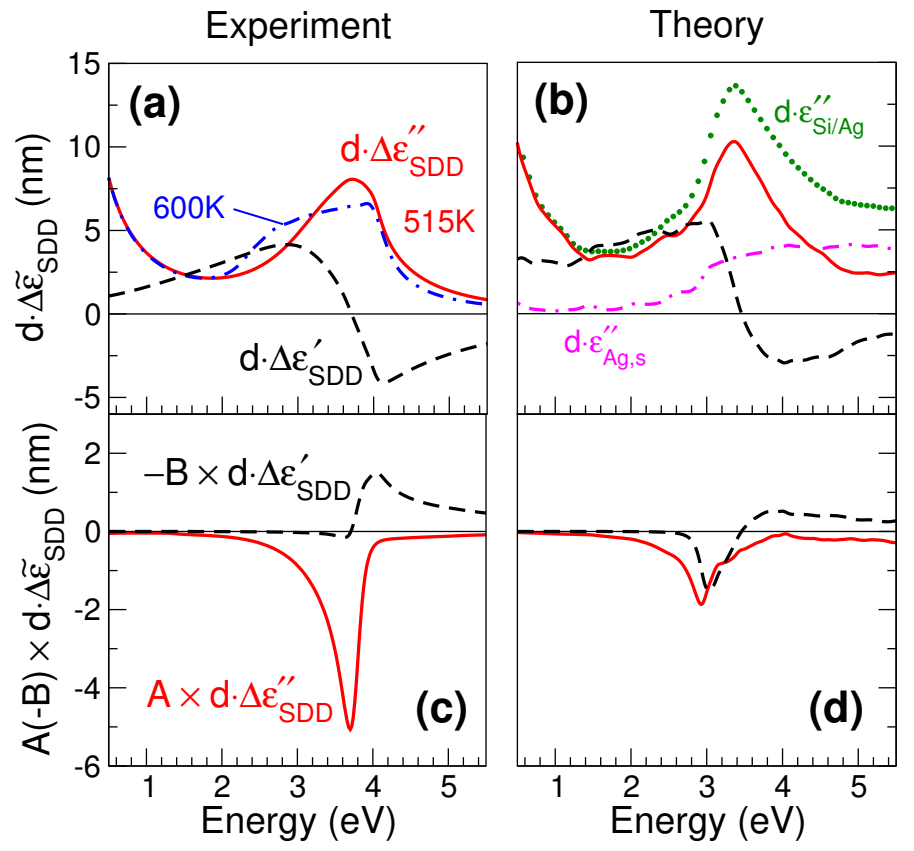

FIG. 12. Surface dielectric difference (SDD) spectra of $\mathrm{Si} / \mathrm{Ag}(111)$. The colour scheme is consistent with Fig. 9. (a) Experimental data at $515 \mathrm{~K}$ and $600 \mathrm{~K}$ (imaginary part only, blue dot-dashed line), showing the influence of the growth temperature on the phase purity. (b) Computed SDD spectra for the $4 \times 4$ structure (solid red and black dashed curves). Also shown are the imaginary parts of the slab dielectric function for the clean (Ag,s: dot-dashed magenta line) and Sicovered surface (Si/Ag: green dots). Panels (c) and (d): as (a) and (b), but including the $A$ and $-B$ prefactors as they appear in Eq. 4. X- and Y-scales are the same for (a) and (b), and for (c) and (d), respectively.

is also reported for growth at $515 \mathrm{~K}$. It exhibits an additional feature at $3.6 \mathrm{eV}$ that is related to the excitation of surface plasmons on $\mathrm{Ag}$ due to the roughening of the surface for this coverage.

As noted in Sec. II B, the $515 \mathrm{~K}$ surface is a mixture of $30 \% 4 \times 4$ phase plus $70 \% \sqrt{13} \times \sqrt{13}$ phase, while the $600 \mathrm{~K}$ surface is dominated by a $2 \sqrt{3} \times 2 \sqrt{3} \mathrm{R} 30^{\circ}$ phase. The similarity between the experimental spectra for different temperatures is reflected in the similarity between the computed dielectric functions for the various phases reported in Cinquanta and coworkers. ${ }^{28}$ Thus in computing the SDRS we consider only a single system, the classic $(4 \times 4)$ phase. Its structural model, shown in Fig.1(c), was proposed by Vogt et al. ${ }^{9}$ and found to be stable across a wide range of $\mathrm{Si}$ chemical potential. ${ }^{11}$ The computed SDRS is shown in Fig. 11(b). Its lineshape and intensity are in reasonable agreement with experiment, although the energy of the negative peak clearly exhibits a redshift of about $0.7 \mathrm{eV}$. As noted previously, this redshift derives from the neglect of many-body effects and the use of purely interband surface dielectric functions.

These spectra are re-analysed in terms of the surface dielectric difference $\Delta \tilde{\varepsilon}_{\text {SDD }}$ (see Eq. 7 ) by inverting Eq. 4 in order to remove the influence of the bulk $A$ and $B$ terms following the process detailed in Sec. IIB. Results for $d \cdot \Delta \varepsilon_{\mathrm{SDD}}^{\prime \prime}$ are presented in Fig. 12(a) for the two growth temperatures. Both spectra are dominated by a broad peak between 3 and $4 \mathrm{eV}$, and their similarity again demonstrates that the influence of the actual mixture of silicene phases is weak. The theoretical SDD spectra (real and imaginary parts) for the $(4 \times 4)$ epitaxial silicene overlayer are reported in panel (b). They are in good agreement with the experimental curves, both in intensity and position. Also shown in the figure are the imaginary parts of the dielectric functions for the bare silver surface $d \cdot \varepsilon_{\mathrm{Ag}, \mathrm{s}}^{\prime \prime}$ and for the $\mathrm{Si} / \mathrm{Ag}$ surface $d \cdot \varepsilon_{\mathrm{Si} / \mathrm{Ag}}^{\prime \prime}=d\left(\varepsilon_{\mathrm{Ag}, \mathrm{s}}^{\prime \prime}+\Delta \varepsilon_{\mathrm{SDD}}^{\prime \prime}\right)$. These data demonstrate that the most pronounced peak in $d \cdot \Delta \varepsilon_{\mathrm{SDD}}^{\prime \prime}$ near $3.5 \mathrm{eV}$ is due to the silicene overlayer. Fig. 12(c) and (d) shows the SDD components multiplied by the $A$ and $-B$ terms: their sum explains the final SDRS lineshape shown in Fig. 11.

These analyses demonstrate that the main SDRS spectral features of $\mathrm{Si} / \mathrm{Ag}(111)$ can once again, like the $\mathrm{Si} / \mathrm{Ag}(110)$ RAS, be traced back to the $\mathrm{Ag}(111)$ substrate via the bulk silver $A$ and $B$ terms (Eq. 5). The negative peak at $3.7 \mathrm{eV}$ in the (experimental) SDRS comes from the negative $A$ peak multiplied by $d \cdot \Delta \varepsilon_{\mathrm{SDD}}^{\prime \prime}$, whereas the positive peak at $4.1 \mathrm{eV}$ in the SDRS comes from the $-B$ peak multiplied by $d \cdot \Delta \varepsilon_{\mathrm{SDD}}^{\prime}$ (see Eq. 4 ). It is interesting to note that, while the agreement between theory and experiment is only reasonable for the whole SDRS signal (compare Fig. 11(a) and (b)), and even somewhat poor for the two main components (Fig. 12(c) and (d)), the comparison for the overlayer itself, after removal of the bulk contribution, is excellent (Fig. 12(a) and (b)). In other words, the optical signal of an epitaxial silicene overlayer on a silver substrate can only be deduced by performing a decomposition of the SDRS spectrum in terms of the SDD, as the lineshape of the SDRS is dominated by bulk silver features.

\section{FREESTANDING SILICENE}

Finally, we consider the optical properties of silicene itself. Freestanding, honeycomb silicene (Fig. 1(a)) is characterized by a calculated Si-Si distance of $a=2.23 \AA$, a sheet buckling of $\Delta=0.45 \AA$, and a Fermi velocity of the electrons of about $0.53 \times 10^{6} \mathrm{~m} / \mathrm{s}$ within DFT-LDA, and $0.65 \times 10^{6} \mathrm{~m} / \mathrm{s}$ after inclusion of quasiparticle effects in the HSE06 approximation. ${ }^{64}$ As discussed in the previous section, when grown epitaxially on $\mathrm{Ag}(111)$ as a $(3 \times 3)$ overlayer the silicon atoms rearrange as a symmetric distribution of flat and outward buckled Si atoms (Fig. 1(c)).

For normal incidence optical properties of an isolated silicene sheet are computed in a similar way as those for surfaces. The 2D objects are simulated by a superlattice arrangement of the silicene sheets. In the limit of independent particles, a formula similar to Eq. 8 de- 


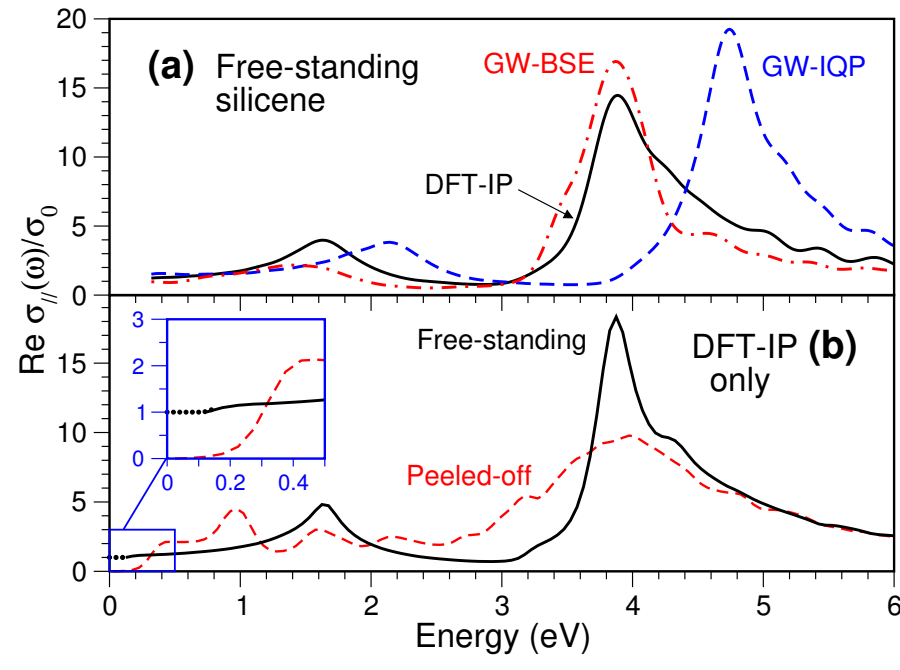

FIG. 13. Real part of the optical conductivity of a silicene sheet computed at (a) different levels of theory: DFT-IP/RPA (black line); GW-IQP (blue dashed line); GW-BSE (red dashdotted line); and (b) for different geometries, within DFT-IP: freestanding (black line), recalculated using a dense k-point grid; and peeled-off the $\mathrm{Ag}(111)$ substrate (red dashed line). The inset highlights the extrapolated data from $0-0.12 \mathrm{eV}$ (dots). The spectra are renormalized to the DC optical conductivity $\sigma_{0}=e^{2} / 4 \hbar$.

scribes the optical absorption. Since the thickness $L$ in Eq. 8 has to be identified with the superlattice period, the quantity $\operatorname{LIm}[\varepsilon(\omega)]$ is independent of the lattice constant $L$ of the artificial superlattice. The optical conductivity $\sigma=-i \omega L \varepsilon(\omega) / 4 \pi$ is, however, a better welldefined quantity to characterize the optical properties of two-dimensional objects. For freestanding silicene, its real part (normalized to the DC quantum optical conductivity $\left.\sigma_{0}=e^{2} / 4 \hbar\right)$ is displayed in Fig. 13(a) in three different approximations for the many-body effects characteristic in excited states. The spectral variation for vanishing frequencies $\omega \rightarrow 0$ are not displayed because of the need of too many $\mathbf{k}$-points in the many-body calculations and the possible appearance of new spectral features of the excitonic insulator phase. ${ }^{65}$ The k-point sampling is crucial. ${ }^{37}$ Too few $\mathbf{k}$-points lead to optical spectra with an artificial fine structure. ${ }^{66}$ Since the perturbative treatment of the quasiparticle corrections only influences the energy eigenvalues, the spectrum in the GW-IQP approximation computed starting from the DFT-LDA eigenfunctions and eigenvalues is mainly blueshifted by about $0.8-1.0 \mathrm{eV}$. The most important peaks related to van Hove singularities at the $\Gamma$ and $M$ points in the $\mathrm{BZ}^{67}$ appear at higher photon energies. To include excitonic effects the Bethe-Salpeter equation (BSE) for the optical response function ${ }^{52}$ is solved. The included screened Coulomb attraction between excited electrons and holes leads to a spectral redistribution resulting mainly in redshifts of the GW spectra. Quasiparticle and excitonic effects tend to cancel each other in two dimensions. As a consequence the DFT spectrum is widely recovered.
The high-energy peak at about $4 \mathrm{eV}$ is hardly influenced, while the low-energy peak at about $1.5 \mathrm{eV}$ is slightly reduced in intensity.

Fig. 13(a) shows that the calculated DFT-IP/RPA optical response of silicene is in qualitative and quantitative agreement with the results obtained in the full threestep procedure. Although excitonic effects are indeed important - as one can deduce by comparing the GWIQP and the excitonic (GW-BSE) spectra-they eventually cancel out almost completely. This provides partial justification for our neglect of excitonic effects in the surface-adsorbed silicene systems discussed previously.

The effect of the structural modification of a silicene layer by a substrate is illustrated in Fig. 13(b). In addition to the IP spectrum of freestanding silicene, calculated with a denser k-point grid, the figure also displays the corresponding spectrum for "peeled-off" silicene (Fig. 1(b)). This corresponds to the $3 \times 3$ silicon overlayer epitaxially grown on a $4 \times 4 \mathrm{Ag}(111)$ surface, but peeled off the substrate such that its atomic geometry is conserved. The lower symmetry with respect to freestanding silicene gives rise to a gap of the order of $0.3 \mathrm{eV} .{ }^{10,11}$ Our results are in good agreement with previous calculations also performed at the IP level. ${ }^{28}$

In this peeled-off silicene layer, the two main peaks discussed above are broadened and reduced in intensity. The main feature around $4 \mathrm{eV}$ thus appears at a slightly higher energy than the peak found in the dielectric function of epitaxially-grown silicene (Fig. 12(b)). Quite dramatic modifications however appear in the lowenergy region. A new double-peaked structure appears, because of the disappearance of the Dirac cones at the $K$ points. The opened gap gives rise to an absorption edge at around $0.3 \mathrm{eV}$. The situation for $\omega \rightarrow 0$ is totally different for the freestanding honeycomb silicene. In the latter, due to the linear bands in the Dirac cones at the BZ boundary at $K$ and $K^{\prime}$ the real part of the conductivity exhibits a plateau governed by the Sommerfeld fine structure constant. ${ }^{37,67}$ These cones have to be sampled with an unusually dense k-point set. ${ }^{37}$

These calculations illustrate how optical spectra yield fingerprints of the presence or destruction of the Dirac cones associated with a pure silicene layer. However, it is important to note that the fingerprints of absorption in the infrared range cannot give conclusive results concerning silicene grown on a silver substrate since the reflectivity of silver is close to $100 \%$ in the infrared and almost unchanged with the presence of silicene. ${ }^{33}$

\section{SUMMARY AND CONCLUSIONS}

We have presented a joint experimental-theoretical study of freestanding silicene and silicenelike phases grown on silver surfaces using optical techniques. First, we have outlined how surface differential reflectance spectroscopy and reflectance anisotropy spectroscopy can be re-interpreted in terms of two quantities, the surface di- 
electric anisotropy and surface dielectric difference, which yield more direct information about the response of the silicon adlayer. The optical response of bulk Ag and clean $\mathrm{Ag}(110)$ were computed at the independent particle level using a DFT-pseudopotential approach. Common approximations and technical difficulties that arise in such calculations were discussed and resolved.

Following this, we presented RAS and SDRS measurements of the $\mathrm{Si} / \mathrm{Ag}(110)$ nanoribbon system and compared with calculations for two proposed structural models. We found that the pentamer chain model yields results in good agreement with the measured data. This constitutes a further rejection of a hexagonal silicenelike geometry in the $\mathrm{Si} / \mathrm{Ag}(110)$ system. ${ }^{23-25}$ In spite of the many structural similarities, the zigzag chain model yielded poor agreement with experiment. RAS was found to be more sensitive than SDRS in this respect. Our analysis also revealed that the RAS and SDRS of $\mathrm{Si} / \mathrm{Ag}(110)$ - or indeed, of any system with a silver substrate-largely derive from the response of bulk Ag. In order to extract direct information about the interface itself, a decomposition into the SDA or SDD is required. This was further demonstrated for the case of epitaxial silicene in the $(3 \times 3) /(4 \times 4) \mathrm{Ag}(111)$ geometry. In this case, optical absorption occurs at an energy $(3.7 \mathrm{eV})$ close to the peak in the reflectivity of the $\mathrm{Ag}$ substrate $(3.9 \mathrm{eV})$, showing that interpretation of optical reflectance measurements must be handled with extreme care.

Finally, we computed the optical spectra of freestanding silicene, with and without many-body corrections.
Such higher order effects are shown to cancel out, suggesting that independent particle calculations are adequate for describing silicenelike systems. When the silicene geometry is deformed following epitaxial growth on a Ag substrate, it becomes semiconducting and the distinct optical signal of the Dirac cones is destroyed. Our results thus provide further confirmation that the optical properties of freestanding silicene are not preserved when deposited on a silver substrate.

In conclusion, we have demonstrated that well-known surface-sensitive optical spectroscopies combined with appropriate $a b$ initio calculations can help to understand the electronic and structural properties of $2 \mathrm{D}$ crystals grown on substrates. Our joint theoretical-experimental approach lead to novel results, i.e. clear optical fingerprints of epitaxial silicene in the $(3 \times 3) /(4 \times 4)$ phase and destruction of its Dirac cones, as well as independent confirmation of the pentamer chain reconstruction of the $\mathrm{Si} / \operatorname{Ag}(110)$ surface.

We have also presented a detailed theoretical approach for computing and analysing RAS and SDRS of 2D overlayers on substrates. We envisage that this approach can be further developed and applied as a non-invasive means for studying current and emerging nanostructured materials of technological relevance.

\section{ACKNOWLEDGMENTS}

$\mathrm{CH}$, OP and PG acknowledge supercomputing support from CINECA through the ISCRA initiative. OP thanks the EU for funding through the HORIZON2020 MSCA RISE "CoExAN" (GA 644076).
* conor.hogan@ism.cnr.it

† olivia.pulci@roma2.infn.it

¥ paola.gori@uniroma3.it

$\S$ Friedhelm.Bechstedt@uni-jena.de

ฯ david.martin@liverpool.ac.uk

** yves.borensztein@sorbonne-universite.fr

1 J. Zhao, H. Liu, Z. Yu, R. Quhe, S. Zhou, Y. Wang, C. C. Liu, H. Zhong, N. Han, J. Lu, Y. Yao, and K. Wu, Progress in Materials Science 83, 24 (2016).

2 C. Grazianetti, E. Cinquanta, and A. Molle, 2D Materials 3, 12001 (2016).

${ }^{3}$ H. Oughaddou, H. Enriquez, M. R. Tchalala, H. Yildirim, A. J. Mayne, A. Bendounan, G. Dujardin, M. Ait Ali, and A. Kara, Progress in Surface Science 90, 46 (2015).

4 M. Houssa, A. Dimoulas, and A. Molle, Journal of Physics: Condensed Matter 27, 253002 (2015).

5 A. Kara, H. Enriquez, A. P. Seitsonen, L. Lew Yan Voon, S. Vizzini, B. Aufray, and H. Oughaddou, Surface Science Reports 67, 1 (2012).

${ }^{6}$ L. Tao, E. Cinquanta, D. Chiappe, C. Grazianetti, M. Fanciulli, M. Dubey, A. Molle, and D. Akinwande, Nature Nanotechnology 10, 227 (2015).

7 C.-C. Liu, W. Feng, and Y. Yao, Physical Review Letters 107, 076802 (2011).
8 L. Matthes, O. Pulci, and F. Bechstedt, Journal of Physics: Condensed Matter 25, 395305 (2013).

9 P. Vogt, P. De Padova, C. Quaresima, J. Avila, E. Frantzeskakis, M. C. Asensio, A. Resta, B. Ealet, and G. Le Lay, Physical Review Letters 108, 155501 (2012).

10 P. Gori, O. Pulci, F. Ronci, S. Colonna, and F. Bechstedt, Journal of Applied Physics 114, 113710 (2013).

11 P. Pflugradt, L. Matthes, and F. Bechstedt, Physical Review B 89, 035403 (2014).

12 J.-I. Iwata, Y.-i. Matsushita, H. Nishi, Z.-X. Guo, and A. Oshiyama, Physical Review B 96, 235442 (2017).

13 A. Curcella, R. Bernard, Y. Borensztein, A. Resta, M. Lazzeri, and G. Prevot, Physical Review B 94, 165438 (2016).

14 H. Jamgotchian, Y. Colignon, N. Hamzaoui, B. Ealet, J. Y. Hoarau, B. Aufray, and J. P. Bibérian, Journal of Physics: Condensed Matter 24, 172001 (2012).

15 D. Chiappe, C. Grazianetti, G. Tallarida, M. Fanciulli, and A. Molle, Advanced Materials 24, 5088 (2012).

16 C.-L. Lin, R. Arafune, K. Kawahara, M. Kanno, N. Tsukahara, E. Minamitani, Y. Kim, M. Kawai, and N. Takagi, Physical Review Letters 110, 076801 (2013).

17 Y. Du, J. Zhuang, J. Wang, Z. Li, H. Liu, J. Zhao, X. Xu, H. Feng, L. Chen, K. Wu, X. Wang, and S. X. Dou, Science Advances 2, e1600067 (2016). 
18 P. M. Sheverdyaeva, S. K. Mahatha, P. Moras, L. Petaccia, G. Fratesi, G. Onida, and C. Carbone, ACS Nano 11, 975 (2017).

19 C. Leandri, G. L. Lay, B. Aufray, C. Girardeaux, J. Avila, M. Dávila, M. Asensio, C. Ottaviani, and A. Cricenti, Surface Science 574, L9 (2005).

20 P. De Padova, C. Quaresima, C. Ottaviani, P. M. Sheverdyaeva, P. Moras, C. Carbone, D. Topwal, B. Olivieri, A. Kara, H. Oughaddou, B. Aufray, and G. Le Lay, Applied Physics Letters 96, 261905 (2010).

21 P. De Padova, P. Perfetti, B. Olivieri, C. Quaresima, C. Ottaviani, and G. Le Lay, Journal of Physics: Condensed Matter 24, 223001 (2012).

22 B. Feng, H. Li, S. Meng, L. Chen, and K. Wu, Surface Science 645, 74 (2016).

23 C. Hogan, S. Colonna, R. Flammini, A. Cricenti, and F. Ronci, Physical Review B 92, 115439 (2015).

24 J. I. Cerdá, J. Sławińska, G. Le Lay, A. C. Marele, J. M. Gómez-Rodríguez, and M. E. Dávila, Nature Communications 7, 13076 (2016).

25 G. Prévot, C. Hogan, T. Leoni, R. Bernard, E. Moyen, and L. Masson, Physical Review Letters 117, 276102 (2016).

26 P. Espeter, C. Keutner, P. Roese, K. Shamout, U. Berges, and C. Westphal, Nanotechnology 28, 455701 (2017).

27 Shaoxiang Sheng, Runze Ma, Jiang-bin Wu, Wenbin Li, Longjuan Kong, Xin Cong, Duanyun Cao, Wenqi Hu, Jian Gou, Jun-Wei Luo, Peng Cheng, Ping-Heng Tan, Ying Jiang, Lan Chen, and Kehui Wu, Nano Letters Article ASAP DOI: 10.1021/acs.nanolett.8b00289

28 E. Cinquanta, G. Fratesi, S. dal Conte, C. Grazianetti, F. Scotognella, S. Stagira, C. Vozzi, G. Onida, and A. Molle, Physical Review B 92, 165427 (2015).

29 P. Weightman, D. S. Martin, R. J. Cole, and T. Farrell, Reports on Progress in Physics 68, 1251 (2005).

30 P. Chiaradia and G. Chiarotti, in Photonic Probes of Surfaces, edited by P. Halevi (Elsevier B.V., Amsterdam, 1995) Chap. 3, p. 97.

31 S. Chandola, K. Hinrichs, M. Gensch, N. Esser, S. Wippermann, W. G. Schmidt, F. Bechstedt, K. Fleischer, and J. F. McGilp, Physical Review Letters 102, 226805 (2009).

32 C. Hogan, E. Ferraro, N. McAlinden, and J. F. McGilp, Physical Review Letters 111, 087401 (2013).

33 Y. Borensztein, G. Prévot, and L. Masson, Physical Review B 89, 245410 (2014).

34 Y. Borensztein, A. Curcella, S. Royer, and G. Prévot, Physical Review B 92, 155407 (2015).

35 G. Prévot, R. Bernard, H. Cruguel, A. Curcella, M. Lazzeri, T. Leoni, L. Masson, A. Ranguis, and Y. Borensztein, physica status solidi (b) 253, 206 (2016).

${ }^{36}$ F. Bechstedt, L. Matthes, P. Gori, and O. Pulci, Applied Physics Letters 100, 2010 (2012).

37 L. Matthes, O. Pulci, and F. Bechstedt, New Journal of Physics 16, 105007 (2014).

38 This is the opposite convention to what is normally found in the literature. However we use it to stay consistent with previous measurements on $\mathrm{Si} / \mathrm{Ag}$ (see Ref.33 and its erratum).

39 J. D. E. McIntyre and D. E. Aspnes, Surface Science 24, 417 (1971).

40 R. J. Cole, B. G. Frederick, and P. Weightman, Journal of Vacuum Science \& Technology A: Vacuum, Surfaces, and Films 16, 3088 (1998).

41 S. Selci, F. Ciccacci, G. Chiarotti, P. Chiaradia, and A. Cricenti, Journal of Vacuum Science \& Technology A:
Vacuum, Surfaces, and Films 5, 327 (1987).

42 S. Colonna, G. Serrano, P. Gori, A. Cricenti, and F. Ronci, Journal of Physics: Condensed Matter 25, 315301 (2013).

43 P. B. Johnson and R. W. Christy, Physical Review B 6, 4370 (1972).

44 E. E. Barritt, PhD thesis, University of Liverpool (2013).

45 P. Moras, T. O. Mentes, P. M. Sheverdyaeva, A. Locatelli, and C. Carbone, Journal of Physics: Condensed Matter 26, 185001 (2014).

46 P. Hohenberg and W. Kohn, Physical Review 136, B864 (1964).

47 W. Kohn and L. J. Sham, Physical Review 140, A1133 (1965).

48 P. Giannozzi, S. Baroni, N. Bonini, M. Calandra, R. Car, C. Cavazzoni, D. Ceresoli, G. L. Chiarotti, M. Cococcioni, I. Dabo, A. Dal Corso, S. de Gironcoli, S. Fabris, G. Fratesi, R. Gebauer, U. Gerstmann, C. Gougoussis, A. Kokalj, M. Lazzeri, L. Martin-Samos, N. Marzari, F. Mauri, R. Mazzarello, S. Paolini, A. Pasquarello, L. Paulatto, C. Sbraccia, S. Scandolo, G. Sclauzero, A. P. Seitsonen, A. Smogunov, P. Umari, and R. M. Wentzcovitch, Journal of Physics: Condensed Matter 21, 395502 (2009).

49 A. Marini, C. Hogan, M. Grüning, and D. Varsano, Computer Physics Communications 180, 1392 (2009).

50 O. Pulci, G. Onida, R. Del Sole, and A. J. Shkrebtii, Physical Review B 58, 1922 (1998).

51 F. Manghi, R. Del Sole, A. Selloni, and E. Molinari, Physical Review B 41, 9935 (1990).

52 F. Bechstedt, Many-Body Approach to Electronic Excitations, Springer Series in Solid-State Sciences, Vol. 181 (Springer Berlin Heidelberg, Berlin, Heidelberg, 2015).

53 http://etsf.polytechnique.fr/exc/.

54 E. D. Palik, ed., Handbook of optical constants of solids (Academic Press, Orlando, 1985).

55 A. Marini, R. Del Sole, and G. Onida, Physical Review B 66, 115101 (2002).

56 Y. Borensztein, W. L. Mochan, J. Tarriba, R. G. Barrera, and A. Tadjeddine, Physical Review Letters 71, 2334 (1993).

57 K. Stahrenberg, T. Herrmann, N. Esser, J. Sahm, W. Richter, S. V. Hoffmann, and P. Hofmann, Physical Review B 58, R10207 (1998).

58 D. S. Martin, N. P. Blanchard, P. Weightman, D. S. Roseburgh, R. J. Cole, J. K. Hansen, J. Bremer, and O. Hunderi, Physical Review B - Condensed Matter and Materials Physics 76, 115403 (2007).

59 W. Mochán, R. Barrera, Y. Borensztein, and A. Tadjeddine, Physica A: Statistical Mechanics and its Applications 207, 334 (1994).

60 S. Bouarab, M. Mebarki, A. Ziane, and M. A. Khan, Physical Review B 63, 195409 (2001).

61 P. Monachesi, M. Palummo, R. Del Sole, R. Ahuja, and O. Eriksson, Physical Review B 64, 115421 (2001).

62 J. Bremer, J. K. Hansen, K. Stahrenberg, and T. Worren, Surface Science 459, 39 (2000).

63 R. Bernard, T. Leoni, A. Wilson, T. Lelaidier, H. Sahaf, E. Moyen, L. Assaud, L. Santinacci, F. Leroy, F. Cheynis, A. Ranguis, H. Jamgotchian, C. Becker, Y. Borensztein, M. Hanbücken, G. Prévot, and L. Masson, Physical Review B 88, 121411 (2013).

64 L. Matthes, O. Pulci, P. Gori, and F. Bechstedt, in Silicene, edited by G. Le Lay and P. Vogt (Springer) Chap. 4. 
${ }^{65}$ D. Jérome, T. M. Rice, and W. Kohn, Physical Review 158, 462 (1967).

${ }^{66}$ W. Wei and T. Jacob, Physical Review B 88, 045203 (2013).
67 L. Matthes, P. Gori, O. Pulci, and F. Bechstedt, Physical Review B 87, 035438 (2013). 